\title{
BIÓPSIA ESTEREOTÃXICA PARA LINFOMAS PRIMÁRIOS DO SISTEMA NERVOSO CENTRAL
}

MURILO S. MENESES*-BRIAN P. O'NEILL*-PATRICK J. KELLY*

\begin{abstract}
RESUMO - Os linfomas primários dơ SNC são raros, mas sua incidência tem aumentado nos últimos anos. Estes tumores frequentemente se localizam nos gânglios da base e tálamo ou se apresentam de forma multifocal. O tratamento atual para eles é a radioterapia, associada ou não a quimioterapia. Devido à sua extremamente baixa morbidade, a biópsia estereotáxica é o método ideal para determinar o diagnóstico históógico em pacientes com suspeita de linfomas do SNC. Os autores apresentam estudo de 49 casos de linfomás primários do SNC, diagnosticado por biópsia estereotáxica.
\end{abstract}

PALAVRAS-CHAVE: linfomas do SNC, biópsia, estereotaxia, tumores intracranianos.

Stereotactic biopsy for primary lymphomas of the central nervous system

SUMMARY - Primary lymphomas of the CNS are rare. However the incidence of these lesions has increased recently. These tumors are often situated in the thalamus and basal ganglia, but can be multifocal. The treatment for primary CNS lymphoma is radiotherapy alone or in association with chemotherapy. Because the associated morbidity is very low stereotactic biopsy is an ideal method for determining the histologic diagnosis in patients with suspected CNS lymphoma. The authors present a study of 49 patients with primary CNS lymphomas in which a stereotactic biopsy was performed.

KEY WORDS: CNS lymphomas, biopsy, stereotaxy, intracranial tumorg.

Os linfomas primários do sistema nervoso central (SNC) são relativamente raros, representando apenas $1,6 \%$ das localizações fora dos linfonodos $8,10 \mathrm{e}$ cerca de $1 \%$ dos tumores intracranianos 10,12,16,21,31. Nos últimos anos tem sido relatado aumento na sua frequência, de forma independente aos casos associados a imunodeficiência, ou induzidos por drogas 27 , ou relacionados com infecção por HIV 7,10,30. Têm sido relatadas regressões espontâneas e claras respostas clínicas e radiológicas ao tratamento somente com corticóides 32 . O principal tratamento utilizado para estes tumores é a radioterapia $4,17,24,25$ e, secundariamente, a quimioterapia 10,26,28. Em princípio, a remoção cirúrgica não traz benefícios, pois são geralmente infiltrantes, multifocais e situados profundamente, ao nível do corpo caloso, dos núcleos da base e região periventricular 17,25. A biópsia estereotáxica é o melhor método para o diagnóstico anátomo-patológico e para orientar o tratamento $1,5,6,14,20,29$. Este procedimento cirúrgico apresenta morbidade extremamente baixa, permite diagnóstico mais preciso, indispensável para o tratamento adequado, e evita cirurgias agressivas e desnecessárias.

Apresentamos 49 casos de linfomas primários do SNC diagnosticados por biópsia estereotáxica.

*Departments of Neurological Surgery and Neurology, Mayo Clinic, Rochester, Minnesota, USA. Dr. Murilo S. Meneses é também Neurocirurgião do Hospital das Nações (Curitiba) e Professor de Neuroanatomia da Universidade Federal do Paraná.

Dr. Murilo S. Meneses - Avenida Getulio Vargas 2159 - 80230 Curitiba PR - Brasil. 


\section{MATERIAL E MÉTODOS}

Entre outubro-1984 e agosto-1991, 49 pacientes foram submetidos a biópsia estereotáxica de tumor intracraniano na Mayo Clinic (Rochester, MN, EUA), com diagnóstico de linfoma primário do SNC. Destes 49 piacientes, 36 eram do sexo masculino e 13 do sexo feminino. A idade dos pacientes variou entre 14 e 83 anos, com média de 56,4 anos. Os mais frequentes sintomas que levaram ao diagnóstico constam da Tabela 1. Nenhum dos pacientes apresentava infeccão por HIV.

Tabela 1. Quadro clínico que levou ao diagnóstico.

\begin{tabular}{lc}
\hline Deficit motor & $28,5 \%$ \\
Quadro demencial & $28,5 \%$ \\
Cefaléia & $12,2 \%$ \\
Afasia & $10,2 \%$ \\
Ataxia & $6,2 \%$ \\
Convulsão & $4,0 \%$ \\
Outros & $10,2 \%$ \\
\hline
\end{tabular}

Biópsia estereotáxica - o aparetho estereotáxico é fixado sob anestesia lccal (sistema COMPASS 13) por 4 pinos de fibra de carbono. São colocadas as marcas de referêricia, em form? de $\mathbf{N}$, para la determinação das coordenadas estereotáxicas pela tomografia axial computadorizada (TAC) e ressonância magnética nuclear (RMN) (Fig. 1). Em certos pacientes é realizada também angiografia cerebral estereotäxica para se localizar os vasos na trajetória da biópsia. O sistema COMPASS permite, pela medida da distância entre os pinos e o aparelho estereotáxico, por micrômetros especiais, a remoção do aparelho quando a bipópsia não é realizada após os exames, sendo recolocado em outro dia. Os cálculos são realizados por computador, comparando-se as coordenadas obtidas por TAC, RMN e, em certos casos, angiografia cerebral. A trajetória é escolhida de modo a não haver lesão vascular ou de tecido cerebral em área funcional. Com as coordenadas do centro do tumor e do ponto de entrada da agulha no crânio, são determınados os ângulos do arco-quadrante no aparelho estereotáxico. O pacienté é colocado na posiçau correspondente à sua lesão. Os tumores da fossa posterior são ábordados em decúbito ventral, utilizando-se o aparelho em posição invertida (Fig. 1). Sob anestesia geral ou local em pacientes mais debilitados, após os cuidados de rotina, é realizada perfuração no crânio e coagulada a dura-máter. introduzida agulha de biópsia de Guiot, com abertura lateral, até o alvo determinadò estereotaxicamente pelas coordenadas nos três planos do espaço. Por aspiração e secção são removidos fragmentos tumorais a cada $10 \mathrm{~mm}$, dependendo do tamanho da lesão. O fechamento é realizado por um ponto de sutura.

\section{RESULTADOS}

Não houve qualquer complicação grave após as biópsias estereotáxicas. Quanto à localizaçāo do tumor, os dados são apresentados na Tabela 2.

Tabela 2. Localização do linfoma no SNC.

\begin{tabular}{lr}
\hline Frontal ou corpo caloso & $28,5 \%$ \\
Tálamo e núcleos da base & $24,4 \%$ \\
Parietal, temporal ou occipital & $20,4 \%$ \\
Fossa posterior & $8,1 \%$ \\
Multifocal & $18,3 \%$ \\
\hline
\end{tabular}

Dos 49 pacientes, 42 foram tratados por radioterapia e, na quase totallidade dos casos a dose fol próxima a 5000 rads. Em 21 pacientes foi utilizada quimioterapia. Em todos os pacientes o exame anátomo-patológico mostrou linfoma primário do SNC. Em 13 deles foi possível diagnostlcar serem tipo células $B$ e em 5 , tipo células $T$. 
Ao rever os prontuários, 25 pacientes tinham falecido. A sobrevida média foi de 15,88 meses. Dos 25, a sobrevida mais curta foi a de um paciente de 56 anos, do sexo masculino, com linfoma de células $T$ parietal esquerdo, que faleceu apenas 11 dias após o diagnóstico.

A sobrevida mais profongada foi de 5 anos e 5 meses: o paciente era um jovem de 17 anos, com linfoma de células $T$ no hemisfério cerebelar esquerdo. Um paciente de 53 anos, com linfoma de células mistas no lobo temporal direito ainda está vivo.
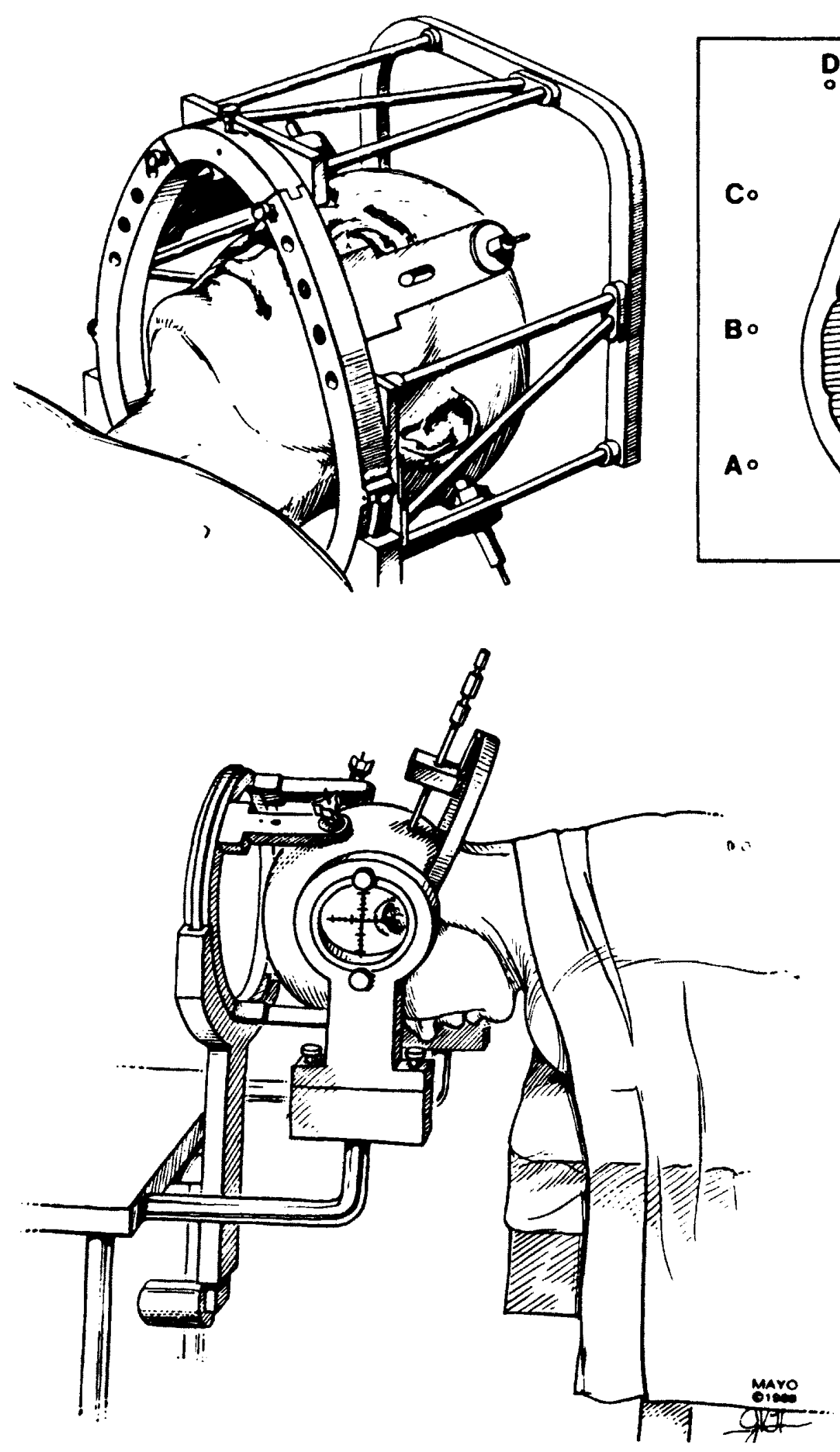

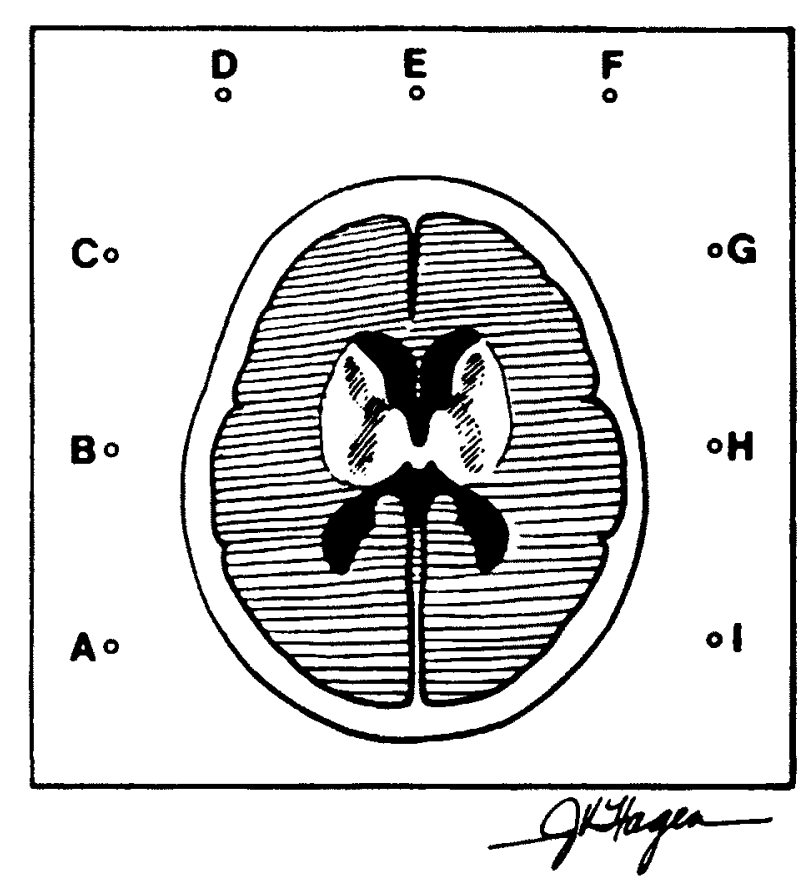

Fig. 1. No alto, paciente com as marcas em $N$ para localizacão estereotáxica na TAC. Em baixo, biopsia da lesão da fossa posterior com o aparelho estereotaxico em posição invertida $e$ o paciente em decúbito ventral.

\section{COMENTARIOS}

Os sinais clínicos dos linfomas do SNC são inespecíficos e estão relacionados à presença de massa intracraniana. Quadro demencial é relatado frequentemente, mas é devido provavelmente à localização da lesão ao nível de corpo caloso e lobos frontais. Os tumores podem se apresentar como lesões únicas ou 
multifocais e envolvem com frequência o tálamo, gânglios da base, lobos frontais e corpo caloso 24 .

Comparando-se à TAC, para neoplasias malignas intracranianas a RMN permite diagnóstico topográfico mais correto, assim como estabelecer a extensão da lesão e sua relação com as estruturas vizinhas $12,23,26$.

Pode existir aspecto cíclico na evolução dos linfomas do SNC, com remissōes espontâneas ou após tratamento com corticóides 32 . Após remissões podem ocorrer recidivas em lugares diferentes das lesões iniciais. $\mathrm{O}$ efeito dos corticóides pode estar relacionado diretamente à ação citotóxica sobre células linfo-reticulares 11. Além disto, a invasão vascular por células neoplásicas parece ser importante fator de alteração clínica, causando oclusões de vasos cerebrais que podem levar a infartos ou hemorragias no tecido tumoral. A tentativa de remover cirurgicamente estes tumores parece não trazer benefícios 24 mas, por se tratar de ato agressivo a lesões profundas do SNC, traria piora do índice de sobrevida 4. O interesse da biópsia estereotáxica é evidente, portanto.

A radioterapia é o tratamento de escolha para os linfomas do SNC. Hochberg e Miller 10 relatam resposta completa ou parcial de $79 \%$ após radioterapia, com aumento de sobrevida para 21,5 meses, o que corresponde a excelente melhora se comparada aos resultados de 1 mês, após unicamente cirurgia 25 . Existe controvérsia quanto à dose de radioterapia porém, devido à frequente localização multifocal, muitos autores acreditam que é necessária a irradiação de todo o encéfalo 24,25. Loeffler et al.19 relatam dois casos de sobrevida prolongada após irradiação do encéfalo e da medula espinhal, mas outros autores 2,9 aconselham a irradiação da medula espinhal somente quando são encontradas células patológicas no líquido cerebroespinhal. A dose mínima de 50 Gy parece ser aceita por diferentes autores $3,18,25$, podendo se utilizar uma dose menor, $45 \mathrm{~Gy}$, para o cérebro e complementar, sobre a região tumoral, 5 a $10 \mathrm{~Gy} 18$.

A quimioterapia parece ter valor importante no tratamento dos linfomas do SNC 12,19, sendo responsável por sobrevidas mais prolongadas quando associada a radioterapia. Resultados de $78 \%$ de sobrevida a 2 anos de evolução, com tratamento associando radioterapia e quimioterapia 28 , têm sido relatados.

Pacientes com lesões multifocais parecem não ter prognóstico pior que aqueles com lesões únicas 24,28. Muitos dos linfomas primários do SNC são tumores de células $B$. Tumores de células $T$ têm sido diagnosticados também, mas o envolvimento multifocal é encontrado em cerca de $50 \% 29$.

Anteriormente à estereotaxia eram utilizados exames invasivos, como ventrículografias e angiografias. Com a utilização de TAC e, mais recentemente, de RMN para biópsias estereotáxicas 13,14 , o procedimento passou a ser mais preciso e menos agressivo. Com novos métodos computadorizados 15,22 é possível, atualmente, a remoção microcirúrgica orientada estereotaxicamente de tumores cerebrais profundos com morbidade muito baixa.

No diagnóstico diferencial dos linfomas do SNC estão incluídos outros tumores intracranianos, processos inflamatórios e infecciosos, principalmente em pacientes imunodeprimidos. A necessidade do diagnóstico histológico, que conduz ao tratamento adequado, é indispensável. Biópsias por cirurgias convencionais de linfomas do SNC são menos precisas, desnecessárias e acarretam risco maior para pacientes muitas vezes debilitados, que não devem ser tratados por remoção cirúr gica e, sim, radioterapia e ou quimioterapia.

A biópsia estereotáxica, nos dias de hoje, é um meio seguro e apresenta baixa morbidade, sendo o método de escolha para o diagnóstico de linfomas do SNC.

\section{REFERENCIAS}

1. Apuzzo MJL, Chandrasoma PT, Cohen D, Zee CS, Zelman V. Computed imaging stereotaxy: experience and perspective related to 500 procedures applied to brain masses. Neurosurgery 1987, 20:930-937.

2. Ashby MA, Bowen D, Bleehen NM, Barber PC, Freer CE. Primary lymphoma of the central nervous system at Addenbrooke's Hospital, Cambridge. Clin Radiol 1988, 39:173-181.

3. Berry MP, Simpson WJ. Radiation therapy in the management of primary malignant lymphoma of the brain. Int $J$ Radiat Oncol Biol Phys 1981, 7:55-59. 
4. Bogdahn U, Bogdahn S, Mertens HG, Dommasch D, Wodarz R. Wunsch PH, Kuhl P, Richter E. Primary non-Hodgkin's lymphoma of the CNS. Acta Neurol Scand 1986, 73:602614.

5. Davis DH, Kelly PJ, Marsch WR, Kall BA, Goerss SJ. Computer-assisted stereotactic biopsy of intracranial lesions. Appl Neurophisiol 1987, 50:172-177.

6. Feiden W, Bise $K$, Steude U. Diagnosis of primary cerebral lymphoma with particular reference to CT-guided stereotactic biopsy. Virchows Archiv $R$ Pathol Anat. 1990, 417:21-28.

7. Formenti SC, Gill PS, Lean E, Rarick M, Meyer PR, Boswell W, Petrovich Z, Chak L, Levine AM. Primary central nervous lymphoma in AIDS: results of radiation therapy. Cancer 1989, 63:1101-1107.

8. Freeman C, Bery JW, Cutler SJ. Occurence and prognosis of extranodal lymphomas. Cancer 1972, 29:252-260.

9. Gonzalez D, Schuster-Uitterhoeve ALJ. Primary non Hodgkin's lymphomas of the central nervous system: results of radiotherapy in 15 cases. Cancer 1983, 51:2048-2052.

10. Hochberg FH, Miller DC. Primary central nervous system lymphoma. J Neurosurg 1988, 68:835-853.

11. Homo-Delarche F. Glucocorticoid receptors and steroid sensitivity in normal and neoplastic human lymphoid tissues: a review. Cancer Res 1984, 44:431-437.

12. Kawakami $\mathbf{Y}$, Tabuchi $\mathbf{K}$, Ohnishi R, Asari S, Nishimoto A. Primary sistem lymphoma J Neurosurg 1985, 62:522-527.

13. Kelly PJ Stereotactic biopsy and resection of thalamic astrocytomas. Neurosurgery 1989, $25: 185-195$.

14. Kelly PJ, Earnest F, Kali BA, Goerss SJ, Scheithauer B. Surgical options for patients with deepseated brain tumors: computer-assisted stereotactic biopsy. Mayo Clinic Proc $1985,60: 223-229$.

15. Kelly PJ, Kall BA. Goerss SJ, Earnest F. Computer-assisted stereotaxic lasel resection of intra-axial brain neoplasms. $J$ Neurosurg 1989, 64:427-439.

16. Kotasek D, Albertyn LE, Sage RE. A five year experience with central nervous system lymphoma. Med J Aust 1986, 144:229-303.

17. Letendre L, Banks PM, Reese DF, Miller RH, Scanlon PW, Kiely JM. Primary lymphoma of the central nervous system. Cancer 1982, 49:939-943.

18. Littman P, Wang CC. Reticulum cell sarcoma of the brain. Cancer 1975, 35:1412-1420.

19. Loeffler JS, Ervin TJ, Mauch P, Skarin A, Weinstein HJ, Canellos G, Cassady JR. Primary lymphomas of the central nervous system: patterns of failure and factors that influence survival. $J$ Clin Oncol 1985, 3:490-494.

20. Lunsforu LD. Diagnosis and treatment of mass lesions using the Leksell stereotactic system. In: Lunsford LD (ed): Modern Stereotactic Neurosurgery. Boston: Nijhof, 1988, p 145-168.

21. McKinstry CS Intracranial malignant lymphoma. J Neurosurg 1987, 67:476-477.

22. Meneses MS, Kelly PJ. Microcirurgia estereotáxica para remoção radical de cistos colóides do terceiro ventrículo. Arq Bras Neurocir (no prelo).

23. Meneses MS, Thurel C, Mikol J, Ramina R, Maniglia JJ, Arruda Wo, Cophignon J. Esthesioneuroblastoma with intracranial extension. Neurosurgery 1990, 27:813-820.

24. Michalski JM, Garcia DM, Kase E, Erigsby PW, Simpson JR. Primary central nervous system lymphoma: analysis of prognostic variables and patterns of treatment failure. Radiology 1990, $179: 855-860$.

25. Murray K, Kun L, Cox J. Primary malignant lymphoma of the central nervous system: results of treatment of 11 cases and review of the literature. J Neurosurg 1986, 65:600-607.

26. Neuwelt EA, Frenkel EP, Gumerlock MK. Developments in the diagnosis and treatment of primary CINS lymphoma: a prospective series. Cancer 1986, 58:1609-1620.

27. Penn I. Lymphomas complicating organ transplantation. Transplant Froc 1983, 15:2790-2.97.

28. Poilack IF, Lunsford LD, Flickinger JC, Dameshek HL. Prognostic factors in the diagnosis and treatment of primary central nervous lymphoma. Cancer 1989, 63:939-947.

29. Sherman ME, Erozan YS, Mann RB. Stereotactic brain biopsy in the diagnosis of malignan lymphoma. Ann J Clin Pathol 1991, 95:878-883.

30. So IT, Beckstead JH, Davis RL. Primary central nervous system lymphoma in acquired immune deficiency syndrom: a clinical and pathological study. Ann Neurol 1986, 20:566-572.

31. Woodmann R, Shin $K$, Pineo G. Primary non-Hodgkin's lymphoma of the brain. Medicine 1985, 64:425-430.

32. Ymasaki $T$, Kikuchi $H$, Yamashita J, Moritake $K$, Shibamoto $Y$, Paine JT, Shima M, Yamabe $H$. Intracerebral malignant lymphoma with lluctuating regression and spatial evolution. Surg Neurobiol 1990, 34:235-244. 